\title{
Pulsed Fluorescence Measurements of Trapped Molecular Ions with Zero Background Detection
}

\author{
Joseph T. Khoury, Sandra E. Rodriguez-Cruz, and Joel H. Parks \\ The Rowland Institute for Science, Cambridge, Massachusetts, USA
}

\begin{abstract}
Sensitive methods have been developed to measure laser-induced fluorescence from trapped ions by reducing the detection of background scattering to zero levels during the laser excitation pulse. The laser beam diameter has been reduced to $\sim 150 \mu \mathrm{m}$ to eliminate scattering on trap apertures and the resulting laser-ion interaction is limited to a volume of $\sim 10^{-5} \mathrm{~cm}^{3}$ which is $\sim 0.03-0.15$ of the total ion cloud volume depending on experimental conditions. The detection optics collected fluorescence only from within the solid angle defined by laser-ion interaction volume. Rhodamine 640 and Alexa Fluor 350 ions, commonly used as fluorescence resonance energy transfer (FRET) fluorophores, were generated in the gas phase by using electrospray ionization and injected into a radiofrequency Paul trap where they were stored and exposed to Nd:YAG laser pulses at 532 and $355 \mathrm{~nm}$ for times up to $10 \mathrm{~m}$. Fluorescence emitted by these ions was investigated for several trap $q_{z}$ values and ion cloud temperatures. Analysis of photon statistics indicated an average of $\sim 10$ photons were incident on the PMT detector per $15 \mathrm{~ns}$ pulse for $\sim 10^{3}$ trapped ions in the interaction volume. Fluorescence measurements displayed a dependence on trapped ion number which were consistent with calculations of the space charge limited ion density. To investigate the quantitative capability of these fluorescence techniques, the laser-induced fragmentation of trapped Alexa Fluor 350 ions was measured and compared with a rate equation model of the dynamics. Decay of the fluorescence signal as well as the parent ion number compared closely with quantitative predictions of the photofragmentation model. (J Am Soc Mass Spectrom 2002, 13, 696-708) () 2002 American Society for Mass Spectrometry
\end{abstract}

$\mathrm{I}$ on traps provide a controlled environment in which fluorescence measurements can be performed on an ensemble of ions over timescales sufficient to consider small ion numbers and slow reaction rates. Laserinduced fluorescence measurements of trapped ions have been used in spectroscopic studies of atomic structure [1-4], the vibrational spectra of small molecules [5-8], as well as to characterize the dynamic cooling [9] and crystallization of atomic ions [10-12].

This paper introduces techniques that eliminate the detection of laser background scattering on trap apertures and internal surfaces allowing measurements to achieve both high sensitivity and large dynamic range. In previous pulsed laser measurements of molecular fluorescence collected from trapped species (discussed in references [5-8]), the radiative lifetimes were sufficiently long to allow collection of fluorescence radiation after the laser pulse. This greatly simplified methods to reduce the detection of background resulting from scattered laser radiation. However, experiments de-

Published online April 23, 2002

Address reprint requests to Dr. J. H. Parks, Rowland Institute for Science, 100 Edwin H. Land Boulevard, Cambridge, MA 02142, USA. E-mail: parks@rowland.org scribed in this paper apply the Nd:YAG pulsed laser to excite strongly emitting molecules having fluorescence lifetimes shorter than the laser pulse. This required the reduction of background laser scattering during laser excitation, a more demanding constraint.

Techniques described in this paper will help to extend fluorescence measurements as an in situ probe of trapped ion properties including chemical identity, the growth and decay of specific ion populations, ion spectroscopy. Fluorescence emission from trapped ions also present an opportunity to quantify conformational changes of biopolymer species in the gas phase [13]. The extension of FRET, widely used in solution studies [14] to measure trapped biopolymer ions can provide the capability to directly correlate changes in fluorescence intensity with changes in the average conformation of biopolymer molecules. For example, FRET methods might be applied to further investigate gas phase dissociation dynamics of double strand oligonucleotide anions [15]. In this case, the rf trap offers a unique capability to correlate the in situ fluorescence data with ion mass spectra which could lead to a new approach to study the intermediate states preceding dissociation of the double strand.

Fluorescence measurements were performed on 


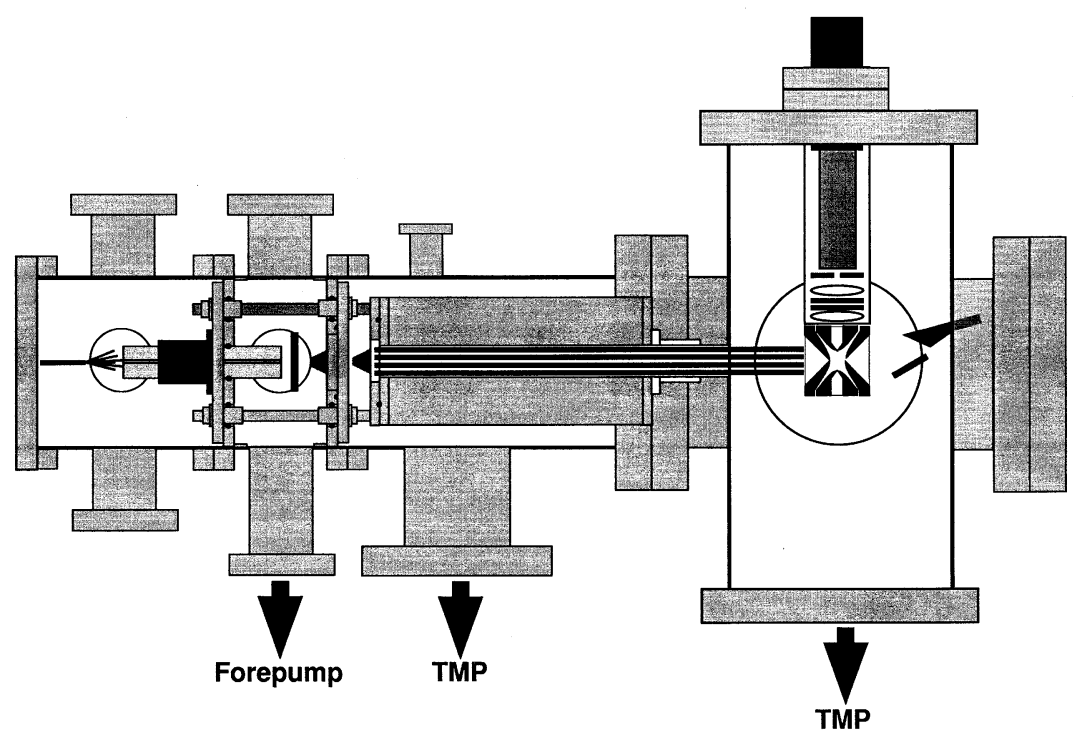

Figure 1. Schematic of the ESI/ion trap/laser-induced fluorescence assembly showing the spray, heated capillary, shutter, two skimmers, $30 \mathrm{~cm}$ octapole ion guide leading into the Paul trap, and finally the ion detector; the distance from the spray to the trap center is $44 \mathrm{~cm}$. Shown above the trap is the (xyz) translatable fluorescence detection apparatus including two lenses, filters, $1 \mathrm{~mm}$ aperture, and PMT.

Rhodamine 640 and Alexa Fluor 350 cations in an rf quadrupole trap. These molecules exhibit strong radiative transitions having short lifetimes of $\tau_{f} \sim 3 \mathrm{~ns}$ characteristic of FRET candidate species. A pulsed laser source was used to excite fluorescence in these experiments; however, the excitation/detection optical configurations would be applicable for excitation by continuous sources as well. These experiments have achieved high sensitivity, zero background measurements of fluorescence through a design of excitation and detection optics configurations that optimized the laserinduced fluorescence from the trapped ion cloud. Measurements were conducted as a function of trap parameters and ion cloud temperature to understand the fluorescence intensity dependence on ion number. In addition, the photofragmentation [16, 17] of Alexa Fluor 350 ions was studied to evaluate the capability of these techniques to obtain quantitative measurements of ion dynamics.

\section{Experimental}

\section{ESI-rf Trap Instrumentation}

Rhodamine 640 (Exciton, Inc., Dayton, $\mathrm{OH}$ ) and Alexa Fluor 350 (Molecular Probes, Inc., Eugene, OR) cations were produced by an electrospray ionization [18-20] source of in-house design and fabrication. A diagram of the electrospray source, $\mathrm{rf}$ trap and fluorescence detection configuration is shown in Figure 1. Rhodamine 640 perchlorate was sprayed from a $2 \times 10^{-5} \mathrm{M}$ methanol solution, and Alexa Fluor 350 carboxylic acid, succinimidyl ester from a $1 \times 10^{-4}$ methanol/water (50:50) solution. The structures of these molecules are shown in Figure 2. Solutions were delivered to the electrospray needle through a fused silica capillary line (100 $\mu \mathrm{m}$ i.d.) at a flow rate of $2 \mu \mathrm{L} / \mathrm{min}$ using a syringe pump (Harvard Apparatus, Holliston, MA). A stainless steel zero dead volume capillary mini-union (Scientific Instrument Services, distributor, manufactured by SGE International, Victoria, Australia) provided electrical contact between the solution and the Al-clad fused silica capillary tubing (Scientific Instrument Services) employed as the electrospray needle (100 $\mu \mathrm{m}$ i.d.). The electric field necessary for electrospray was obtained by applying a high voltage $(2.2-3.8 \mathrm{kV})$ to the capillary mini-union.

Hydrated ions were sprayed directly into a $0.5 \mathrm{~mm} \times$ $20 \mathrm{~cm}$ stainless-steel capillary embedded in a cylindrical, copper block which is heated to $125^{\circ} \mathrm{C}$ by cartridge heaters (200W, Watlow, St. Louis, MO) to remove excess water. Ions emitted from the capillary are directed through a solenoid driven shutter followed by two skimmers $(0.5 \mathrm{~mm}$, BEAM Dynamics, San Carlos, CA) and propagated through a $30 \mathrm{~cm}$ octapole ion

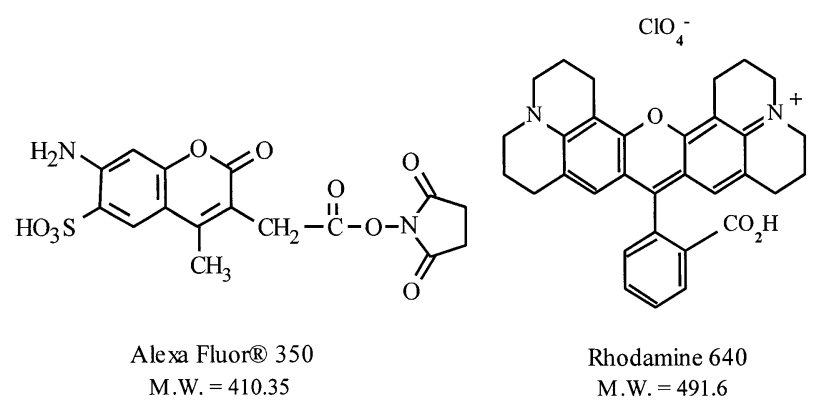

Figure 2. Structures of the molecules used in these fluorescence experiments. The molecular weight shown for Rhodamine 640 does not include the perchlorate ion. 
guide $\left(r_{0}=2.4 \mathrm{~mm}\right)$ to the grounded endcap electrode of an $\mathrm{rf}$ ion trap assembly. Ions are injected through a $1.2 \mathrm{~mm}$ diameter aperture in the end-cap. The volume after the capillary is pumped by a $10 \mathrm{~L} / \mathrm{s}$ forepump (Leybold, Vacuum, Cologne, Germany) and a $170 \mathrm{~L} / \mathrm{s}$ turbomolecular pump (Varian Vacuum Technologies, Torino, Italy) evacuates the volume between the skimmers.

The rf ion trap [21] was designed and fabricated in-house with an end-cap electrode spacing of $6 \mathrm{~mm}$ $\left(z_{0}=3 \mathrm{~mm}\right)$ and operates at an $\mathrm{rf}$ drive frequency of $600 \mathrm{kHz}$. All experiments were performed without bias voltage on the ring electrode $\left(a_{z}=0\right)$. The trap vacuum chamber is pumped by a $600 \mathrm{~L} / \mathrm{s}$ turbomolecular pump (Pfeiffer Vacuum, Nashua, NH). Helium was admitted directly into the trap to maintain a constant chamber pressure of $5 \times 10^{-6}$ torr during ion storage. The $\mathrm{He}$ pressure was pulsed to $2 \times 10^{-4}$ torr during ion injection which varied between $0.25 \mathrm{~s}-5 \mathrm{~s}$. The trap assembly and He background gas are both temperature controlled to enable collisional cooling of the trapped ions to $\sim 140 \mathrm{~K}$ and heating to $\sim 150{ }^{\circ} \mathrm{C}$. After exposure to laser excitation, the mass spectrum and number of the stored ions was obtained by ejecting the ions through the endcap electrode into an external dynode electron multiplier (model 7596M, KM Electronics, Tucker, GA). Electronics were designed to measure the rate of ions detected which is then converted into the number of ions incident on the electron multiplier during the ion ejection time. The number of trapped ions are estimated to be a factor of $\sim 2$ times the number of detected ions. In these experiments the trapped ion number was varied over the range $10^{3} \leq N \leq 6 \times 10^{4}$. Trap voltages required for ion manipulation and data acquisition were controlled by LabView software (National Instruments Corp., Austin, TX).

The trapped ion cloud is excited with the higher harmonics of a pulsed Nd:YAG laser $(\sim 15 \mathrm{~ns}$ pulselength, $10 \mathrm{~Hz}$ repetition rate) which have been generated by a KD*P nonlinear crystal (Inrad, Northvale, NJ) at 532,355 , or $266 \mathrm{~nm}$. The beam is spatially filtered by $f=3.2 \mathrm{~cm}$ lenses and a $50 \mu \mathrm{m}$ pinhole before being focused to a waist size of $\sim 150 \mu \mathrm{m}$ by a $50 \mathrm{~cm}$ focal length UV grade quartz lens. Since the Rayleigh range is much longer than the distance between ring apertures, the waist size closely estimates the interaction cross section with the ion cloud. The overlap or interaction volume of the focused laser beam and the ion cloud is estimated to be $\sim 3-15 \%$ of the total volume of the ion cloud depending on experimental conditions such as ion cloud temperature, trap operating point $\left(q_{z}\right)$, and number of trapped ions.

The laser beam entered and exited the trap through $1.2 \mathrm{~mm}$ apertures in the ring electrode and fluorescence was detected at $90^{\circ}$ to the beam axis. The laser polarization was maintained perpendicular to the detection axis for each harmonic used to optimize fluorescence detection. Both the exit and entrance windows of the high-vacuum chamber were positioned at Brewster's angle to minimize reflections and scattering of laser light into the detection solid angle. The window ports were also baffled to reduce the laser light scattered by the last optical element from entering the chamber. Laser pulse energies of $\leq 3 \mu \mathrm{J}$ (intensity of $\leq 1 \mathrm{MW} /$ $\mathrm{cm}^{2}$ ) were used to excite ion fluorescence in these experiments. The fluorescence intensity was measured for each molecule as a function of laser intensity; and for pulse energies $\leq 3 \mu \mathrm{J}$ the fluorescence increased approximately linearly.

As shown in Figure 1, the fluorescence emission was collected through a third $1.2 \mathrm{~mm}$ aperture in the ring electrode by a pair of optical grade quartz lenses that focused the cloud emission on a $1 \mathrm{~mm}$ pinhole placed in front of the photomultiplier (R1463, Hamamatsu Photonics, Hamamatsu, Japan). This arrangement imaged the center of the ion cloud fluorescence on the detection aperture which reduced the amount of scattered light reaching the PMT which originated outside the lasercloud interaction volume. The detection solid angle was limited by the trap aperture to $\Delta \Omega / 4 \pi \sim 5 \times 10^{-3}$. For laser excitation of Rhodamine 640 at $532 \mathrm{~nm}$ and Alexa Fluor350 at $355 \mathrm{~nm}$, background scattering was further reduced by using two dielectric high reflectors having $\mathrm{R}$ $\sim 99 \%$ at the laser wavelength, and several Schott glass long-pass filters (OG550 and GG395 respectively) which transmit throughout the fluorescence bandwidth. Excitation at $355 \mathrm{~nm}$ required an additional dichroic longpass filter (o.d. $\sim 6$ at the laser wavelength). The choice of bandpass filters used to isolate the fluorescence of interest requires an appreciation that the fluorescence can blue shift in vacuum relative to solution measurements. In particular, this shift is considerable $(\sim 30 \mathrm{~nm})$ for Xanthene dyes [22] and completely prevented the observation of Rhodamine 6G fluorescence with a filter set based on solution wavelengths.

The detection optics and PMT are positioned within the trap vacuum chamber and are mounted on an (xyz) precision manipulator (Thermionics Vacuum, Port Townsend, WA). Optical alignment of the detector beamline was necessary to peak the fluorescence signal on the central portion of the ion cloud so that the fluorescence data was dominated by the peak cloud density. Scanning the detection optics along the radial $(r)$ and axial $(z)$ ion cloud directions produced the intensity data shown in Figure 3. Fitting these scans to gaussian functions yielded a ratio of the full widths at half maximum of $\sigma_{r} / \sigma_{z} \approx 1.5$. This ratio is less than the expected ratio of cloud dimensions determined by the trap geometry, $\left(r_{0} / z_{0}=2\right.$, as a result of the convolution of the cloud fluorescence by the detection apertures.

The pulsed fluorescence signal from the PMT enters a low noise preamplifier (gain $=25,300-\mathrm{MHz}$ bandwidth, SR445, Stanford Research Systems, Sunnyvale, CA) and then is passed to a gated integrator (Stanford Research Systems, SR250). The integrator output for each laser pulse is input to a computer A/D board controlled by LabView software. 


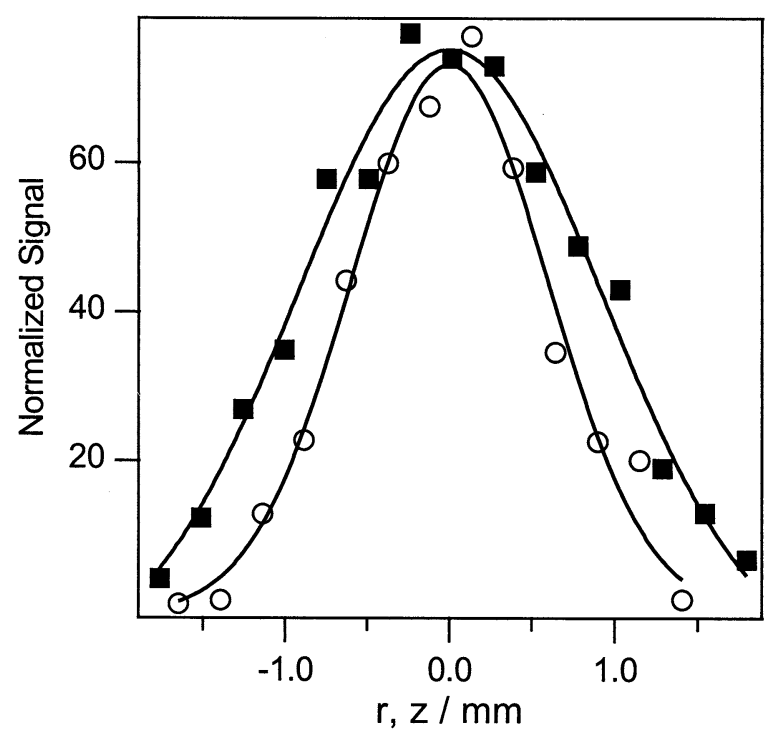

Figure 3. Relative intensity distribution of the emitted fluorescence obtained by scanning the detector assembly (Figure 1) along $\mathrm{r}$ and $\mathrm{z}$. The data points are the fluorescence signal as a function of distance along $r$ (filled square) and $\mathrm{z}$ (open circle); the solid curves are gaussian fits to the data, which describe the convolution of the $1 \mathrm{~mm}$ detector aperture with the emitting volume of the ion cloud.

\section{Results and Discussion}

\section{Photon Statistics}

An example of the fluorescence signals obtained from the boxcar integrator is shown in Figure 4 . Data in Figure 4a are obtained from $\sim 10^{3}$ Rhodamine 640 ions within the interaction volume which were exposed to laser pulses having an intensity of $1.2 \mathrm{MW} / \mathrm{cm}^{2}$ (pulse energy $3.2 \mu \mathrm{J}$, pulse width $15 \mathrm{~ns}$ ) at $532 \mathrm{~nm}$. Ions were trapped at $q_{z}=0.61$ and the trap/He gas temperature was $T=300 \mathrm{~K}$. Each peak represents the photoelectrons produced by fluorescence from a single laser pulse; note that this data shows no evidence of decay over the entire $10 \mathrm{~min}$ exposure. Fluctuations in the number of photoelectrons are observed more clearly in the expanded view of 100 laser shots shown in Figure 4b. Note that a large fraction (almost $40 \%$ in this case) of laser pulses resulted in zero detected photoelectrons. In the absence of trapped ions, Figure $4 c$, the data displays zero background signal.

These data can be described by considering fluctuations in both the number of photons per pulse incident on the PMT and in the resulting number of photoelectrons. Assuming the incident photons are described by a Poisson distribution, the fraction of data pulses resulting in $r$ photoelectrons is derived in the Appendix B as

$$
P_{r}(\eta)=\sum_{m} P_{m} P_{r, m}(\eta)=\frac{\left.(\mu \eta)^{r}\right)}{r !} e^{-\mu \eta}
$$

where $P_{m}$ is the probability that $m$ photons will strike the PMT during a laser pulse and $P_{r, m}$ is the probability that $r$ photoelectrons are produced for $m$ incident photons. The PMT quantum efficiency is $\eta \approx 0.1$ near the
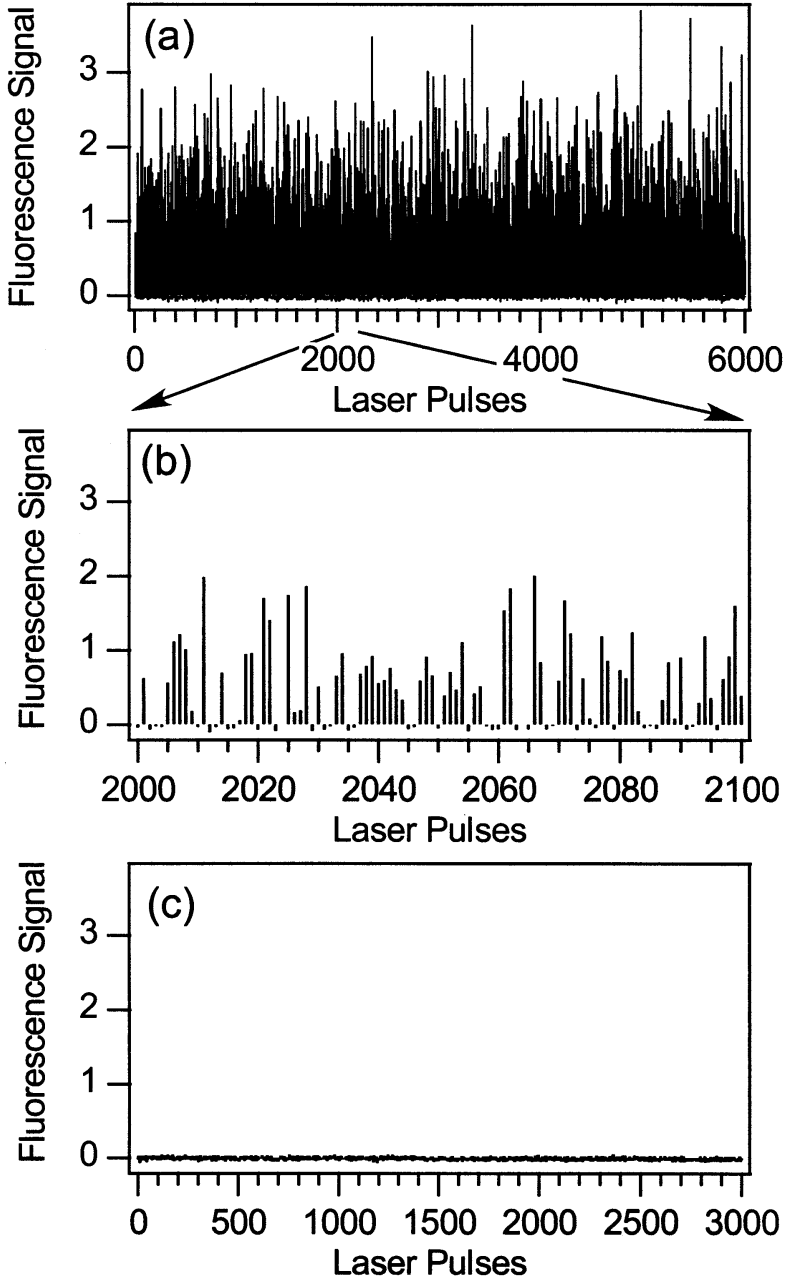

Figure 4. Fluorescence signals with (a) $N=1000$ Rhodamine 640 ions within the interaction volume at $q_{z}=0.61$, and $T=300 \mathrm{~K}$. Each spike represents the fluorescence signal from a single laser pulse at $532 \mathrm{~nm}$. In (b) the expanded view of 100 laser shots shows the statistical fluctuations in the signal. In (c) the absence of fluorescence is shown for $N=0$ ions in the trap (zero background).

peak fluorescence wavelength; and $\mu$ is the average number of photons incident during each pulse. The probability at $r=0, P_{0}(\eta)=e^{-\mu \eta}$, is just the fraction of zeros taken directly from the data, and an average over seven data sets taken under identical conditions yields $\langle\mu\rangle=10 \pm 1$ photons per pulse for $\eta \approx 0.1$. Figure 5 displays a histogram derived from seven data sets taken under identical conditions to that shown in Figure 4. The data bin size was chosen to give a best fit to the calculated distribution, $P_{r}(\eta)$, which is completely determined by the fraction of pulses resulting in $r=0$ and indicated in Figure 5 by the solid squares. Comparison of the calculated photoelectron distribution and the data histogram indicates that the fluctuations in signal intensity closely follow the expected statistical distribution.

The average number of photons incident on the PMT detector for a single laser pulse can also be derived using experimental parameters to evaluate 


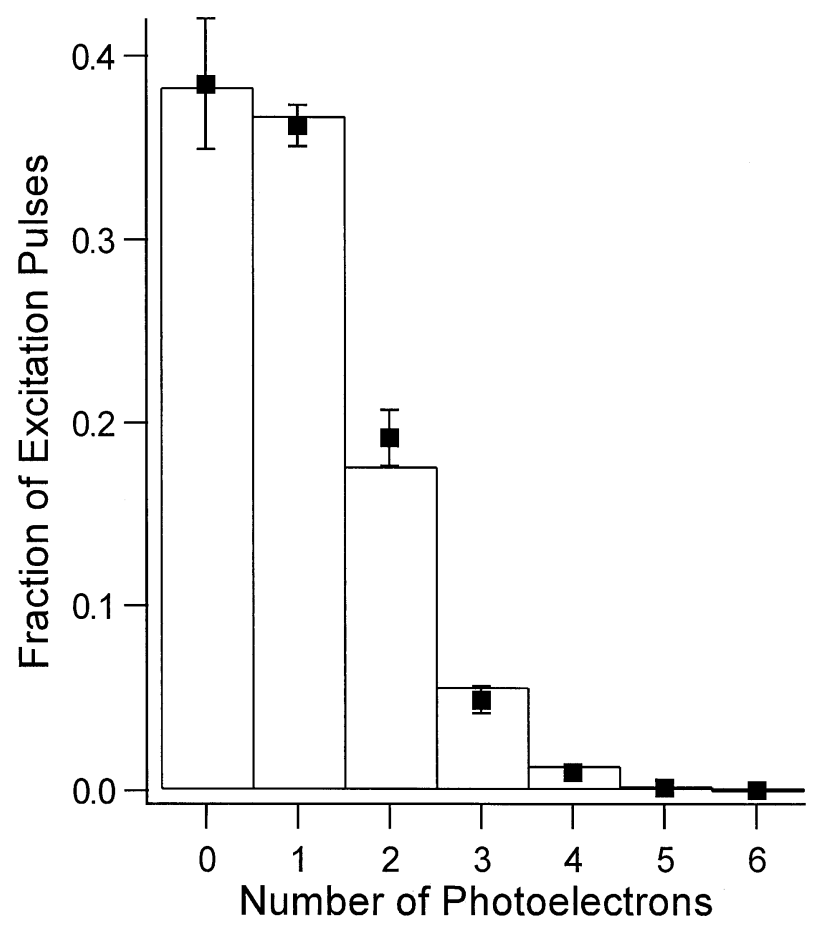

Figure 5. The bar graph plots the photon statistics expected from a Poisson photoelectron distribution (see Appendix B). The solid squares are a histogram of the fluorescence data averaged over seven independent data sets taken under identical conditions.

$$
\left\langle N_{h v}\right\rangle=\left[\frac{n_{p} \delta V \quad I / I_{S} \quad \delta \Omega}{\tau_{f} 1+2\left(I / I_{S}\right) 4 \pi} Q Y\right] \tau_{p} T(1-R)
$$

as shown in Appendix A2. The bracketed term in eq 2 is just the photon emission rate into solid angle $\delta \Omega$. Calculating the space charge limited ion density, $n$, as outlined in the Appendix A1 yields $\left\langle N_{h v}\right\rangle \approx 6$ photons per laser pulse. This value is comparable to that obtained from photon statistics data to within the uncertainty introduced by the interaction volume and ion density $\left(n_{p} \delta V\right)$ of roughly a factor of $\sim 1.5$.

\section{Space Charge Limited Fluorescence}

The total fluorescence intensity was measured for each ion as a function of the detected ion number. These measurements are reported as a sum of the detected signal peaks for a given ion exposure time. Measurements of Rhodamine 640 fluorescence intensity versus detected ion number are displayed in Figure 6a. Each data point was obtained at a pulse energy of $\sim 3 \mu \mathrm{J}$ for an exposure time of $3 \mathrm{~min}$. Data were taken for trap temperatures $\mathrm{T}=140 \mathrm{~K}, 300 \mathrm{~K}$, and trap parameters $q_{z}=0.38,0.61$ (corresponding to well depths of 5.9 and $9.5 \mathrm{eV}$, respectively). The error bars shown in Figure 6 a represent \pm 1 standard deviation for measurements taken over several identical runs. For each parameter pair, the fluorescence signal increases nonlinearly with ion number and tends to saturate. However, the number of trapped ions was observed to increase
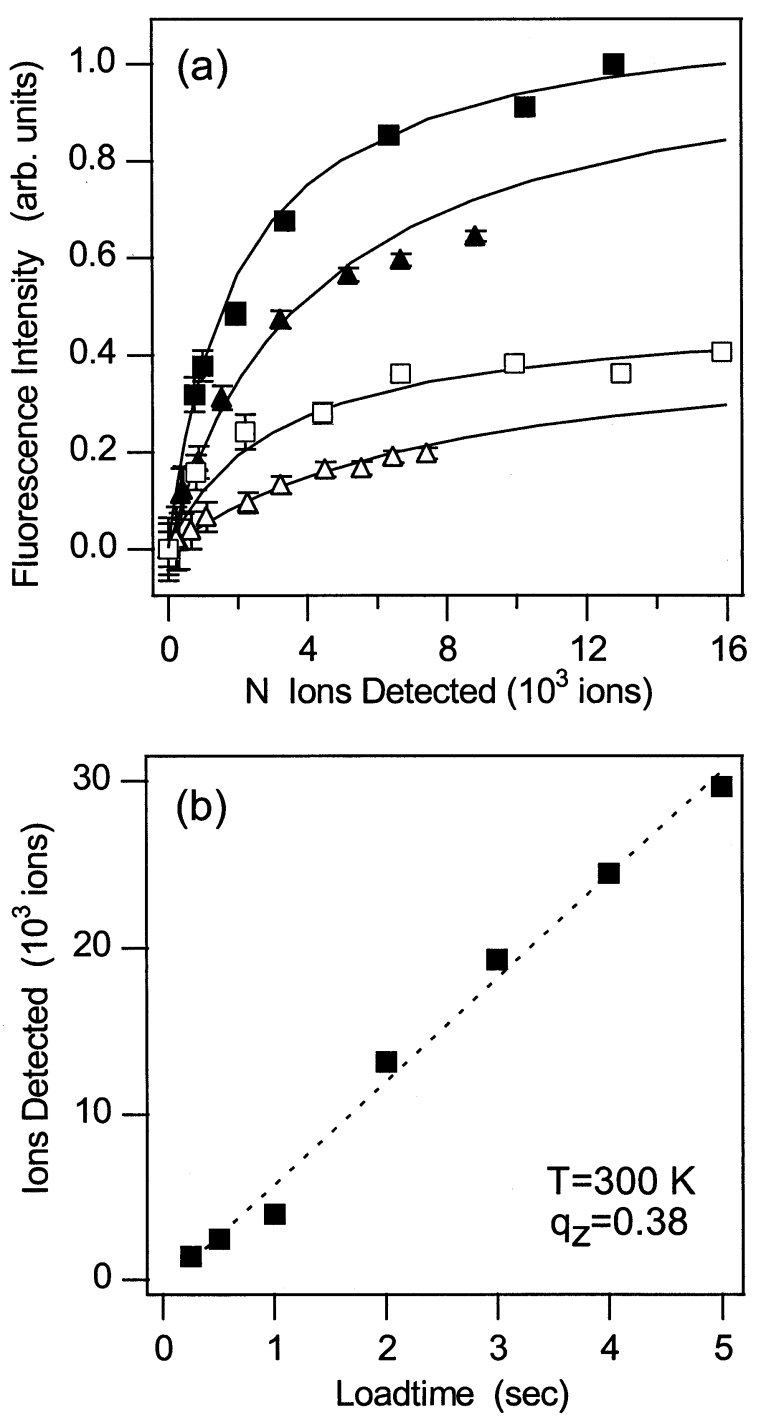

Figure 6. (a) Total fluorescence versus $N$ for various trapping conditions: (filled square) $q_{z}=0.61, \mathrm{~T}=140 \mathrm{~K}$; (filled triangle) $q_{z}=0.61, \mathrm{~T}=300 \mathrm{~K}$; (open square) $q_{z}=0.38, \mathrm{~T}=140 \mathrm{~K}$; (open triangle) $q_{z}=0.38, \mathrm{~T}=300 \mathrm{~K}$. Each point was taken using $\sim 3 \mu \mathrm{J}$ pulse energy at $10 \mathrm{~Hz}$ for $3 \mathrm{~m}$. Error bars represent \pm 1 standard deviation for measurements taken over several identical runs. Solid curves derive from space charge calculations of the ion densities. (b) $N$ versus Ion Load-time is linear under all conditions shown here for $q_{z}=0.38$ and $\mathrm{T}=300 \mathrm{~K}$ as an example.

linearly with loading time, as shown in Figure 6b, which eliminates saturation of the ion loading and detection process as a possibility for this intensity behavior. The fluorescence signal increases for larger trapping well depth and lower kinetic temperature, as displayed by the four sets of data shown in Figure 6a.

The fluorescence intensity is proportional to the density of ions in the interaction volume. The trapped ion cloud distribution, given by

$$
n(r, z)=n_{p} \exp (-e \Phi(r, z) / k T)
$$

where

$$
\Phi(r, z)=\Phi_{T}+\varphi_{s c}
$$


is an ansatz which introduces the space charge potential, $\varphi_{s c}$ to include the effects of ion-ion Coulomb interactions. Only for small ion number is the density linear in $N$, ion number, and accurately approximated in this limit by a trap potential $\Phi_{T}(r, z)$ which is a quadratic function of $r$ and $z$ [23]. As the ion number increases, Coulomb repulsion forces expand the ion cloud and cause the ion density to deviate from a linear dependence on ion number.

Calculations of the ion density in the presence of space charge were performed for each parameter set as outlined in the Appendix A1 and described more fully in references [23] and [24]. The peak density calculations are shown in Figure 6 by solid curves that have been normalized to the intensity data for comparison. In plotting the calculation results, the trapped ion number of the calculation was taken to be exactly twice the detected ion number measured. This assumes that ion loss during ejection into the electron multiplier was not significant. In fact, the calculation for $\left(q_{z}=0.38, T=140\right.$ $\mathrm{K})$ and $\left(q_{z}=0.61, T=140 \mathrm{~K}\right)$ fit the data very closely in this approximation and data for $\left(q_{z}=0.38, T=300 \mathrm{~K}\right)$ and $\left(q_{z}=0.61, T=300 \mathrm{~K}\right)$ required only $5-10 \%$ reductions in the stored ion number to optimize the fit. In each case, the nonlinear increase in fluorescence signal with ion number closely follows the form of the peak density calculation. This strongly suggests the fluorescence increase with ion number is reflecting the density saturation induced by the ion cloud spacecharge forces. As expected, the highest peak density was observed for the deeper well depth $\left(q_{z}=0.61, D_{z} \sim\right.$ $15.3 \mathrm{eV}$ ) and lower kinetic temperature of $140 \mathrm{~K}$.

\section{Photofragmentation}

Fluorescence measurements were also performed for Alexa Fluor 350 which required excitation at $355 \mathrm{~nm}$. The fluorescence displayed characteristics similar to Rhodamine 640 but with an important exception: The Alexa Fluor 350 fluorescence exhibited a decay resulting from photofragmentation of the trapped ions. Rhodamine 640 fluorescence did not decay until the pulse energy was increased to $\sim 6 \mu \mathrm{J}$; however, significant fluorescence decay was observed for Alexa Fluor 350 at a pulse energy of $\leq 2 \mu \mathrm{J}$. Measurements were performed to model the dependence of this fluorescence decay on experimental parameters and postpone a more detailed understanding of energy flow in the molecular process to future investigation.

Analysis of the photofragmentation data relied on the rate equation model derived in the Appendix. In this model, the parent ion number after $m$ laser pulses of intensity $I$ is given by

$$
N_{m}=N_{0} \exp \left(-\Gamma_{F} m\right)
$$

where

$$
\Gamma_{F}=k_{F} \tau_{p} \frac{I / I_{S} \quad \delta V}{1+2\left(I / I_{S}\right) V}
$$

is just the fraction of parent ions fragmented per laser pulse. In eq $6, k_{F}$ is an average photofragmentation rate defined in Appendix $C$; $\tau_{p}$ is the laser pulsewidth; and $m$ is the number of laser pulses. The fragmented ion fraction saturates with increasing laser intensity as the induced transition rate to the ion ground state increases. The saturation intensity, $I_{S}$, for the radiative transition is defined in eq A4 of the Appendix. In the derivation of eq $\mathrm{A} 4$, the ratio of the interaction volume to ion cloud volume, $\delta V / V$, can be approximated by defining the ion cloud volume as $V \equiv N_{m} / n_{p}$. Calculations described in the Appendix indicate that this volume fraction increases slowly as $N_{m}$ decreases as a result of space charge interactions. As shown in Figure $10 \mathrm{~b}, \delta \mathrm{V} / \mathrm{V}$ increases by a factor of $\sim 2$ as $N$ decreases by a factor of 10-15. Note that the decay rate, $\Gamma_{F}$, is significantly reduced by this interaction volume fraction because the unexposed ion density outside the laser-ion cloud interaction volume acts as a reservoir which refills the excitation volume with new, unexposed ions during the $100 \mathrm{~ms}$ between each laser pulse.

The decay of the fluorescence signal resulting from photofragmentation follows from eq A23 of the Appendix for the number of photons $\left\langle N_{h v}\right\rangle$ detected from the interaction volume $\delta \mathrm{V}$

$$
\begin{aligned}
\left\langle N_{h v}\right\rangle & =\text { constant } \cdot n_{e} \delta V \\
& \approx \text { constant } \cdot N_{0} \frac{\delta V}{V} \exp \left(-\Gamma_{F} m\right) .
\end{aligned}
$$

Although the volume fraction $\delta V / V$ will be approximated by a constant in this model in the fit analysis to extract $\Gamma_{F}$ and also to determine $k_{F}$ using eq $6, \delta V / V$ actually varies slowly with the trapped ion number as shown in the Appendix A1. As a result, this approximation will introduce a small uncertainty in $\Gamma_{F}$, and $k_{F}$, the fluorescence decay rate will be expected to differ from the ion decay rate. However, to obtain a consistent comparison between $\Gamma_{F}$ derived from decay of the parent ion and that obtained from the fluorescence data, the ion number dependence of the preexponential factor $\delta V / V$ in eq 7 will have to be included in the fit analysis of fluorescence data.

Parent ion decay. An indication that Alexa Fluor 350 fluorescence decay was associated with photofragmentation was obtained by observing the dependence of ion mass spectra on exposure to laser pulses. Figure 7a displays a decay of the parent ion (mass $\sim 411 \mathrm{a} \mu$ ) abundance with simultaneous growth of an ion fragment (mass $\sim 280 \mathrm{a} \mu$ ) as exposure time increases. The data shown in Figure 7a was taken at $q_{z}=0.46, T=300$ $\mathrm{K}$ with $\sim 2 \mu \mathrm{J}$ pulses and each data point represents the number of ejected ions after exposure to $m$ laser pulses. 

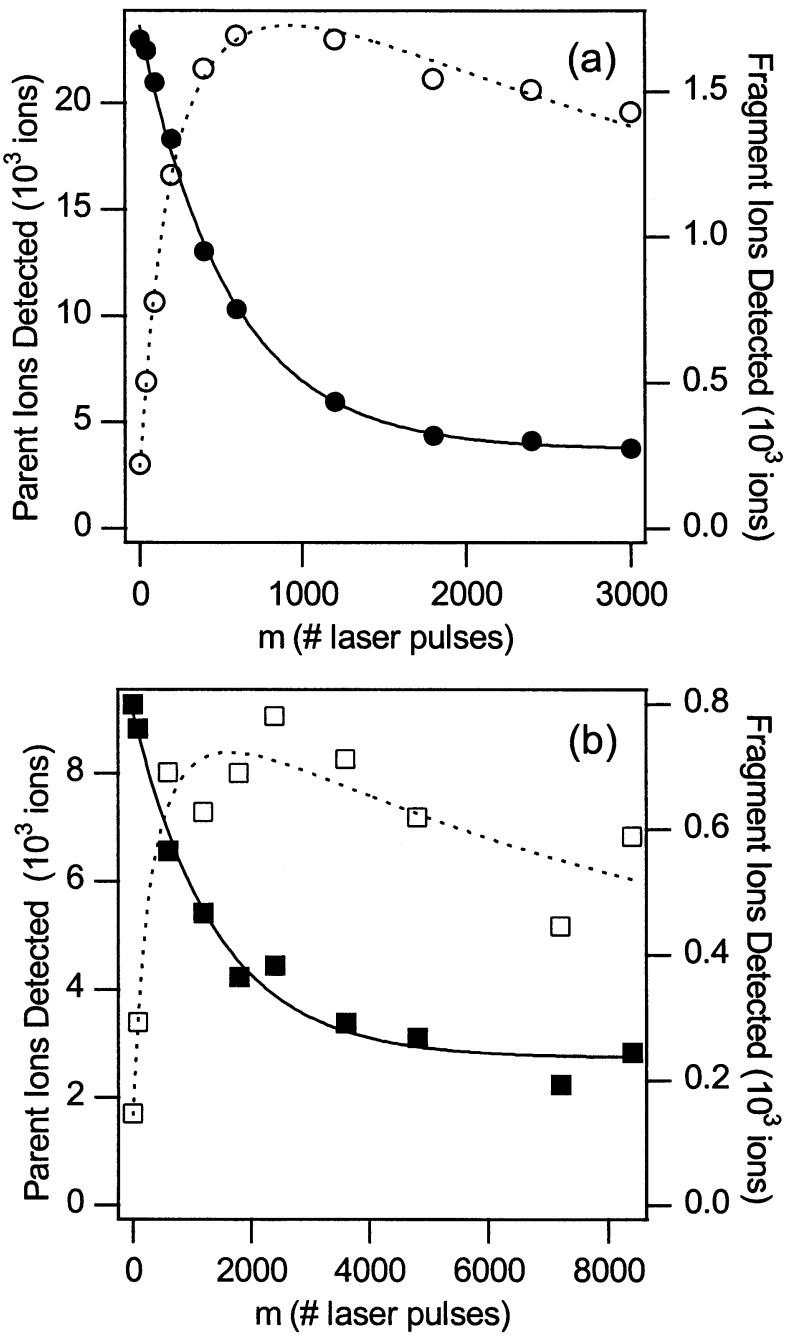

Figure 7. The parent ion number (filled circle, filled square) displays a decrease during exposure resulting from photofragementation, and the associated growth of a fragment ion is also shown (open circle, oprn square). The solid curves are fits to the data as described in the text. Decay rate is higher at room temperature (a) $\mathrm{T}=300 \mathrm{~K}$, than at low temperature (b) $\mathrm{T}=140 \mathrm{~K}$. All data were taken with $2 \mu \mathrm{J} /$ pulse, $q_{z}=0.46$.

The ion fragment abundances were an order of magnitude lower than the parent ion as a result of reduced trap stability for ions of $m \leq 280$ at $q_{z} \geq 0.7$; lighter fragments were also not detected in mass spectra under our experimental conditions. The ion decay was also measured at $T=140 \mathrm{~K}$ for the same trap parameter $q_{z}=0.46$ and is shown in Figure $7 \mathrm{~b}$. Note that the growth of the fragment abundance peaks and then declines as the parent ion decays, which may result from either reduced trap stability of this species or possibly subsequent fragmentation. The decay data was fit to the function $f(m)=a_{0}+a_{1} \exp \left(-\Gamma_{F} m\right)$ where $m$ is the number of pulses. The fraction of parent ions fragmented per laser pulse determined by this fit is listed in Table 1 for both temperatures. The rate constants $k_{F}$ were estimated from eq 6 by taking the volume ratio as the calculated average of $\langle\delta V / V\rangle_{N}$ weighted by ion number; these parameters are also listed in Table 1. Further discussion of these fragmentation rates will follow the next section.

Fitting the ion decay data to this photofragmentation model requires a constant term $a_{0}$. Note that this model represented the ion cloud as a diffusive gas without including the long range correlations describing the trajectory of each trapped ion. Such a model will not be appropriate to describe physical processes which occur predominantly for specific ensembles of trajectories. In this case, those trajectories which do not spend sufficient time within the interaction volume would fragment at a significantly slower rate. The constant term observed in the data could be representing such a reduced decay rate that would be observed after the more readily fragmented ions were eliminated.

Fluorescence signal decay. Decay of the fluorescence signal of Alexa Fluor 350 was measured at at two different laser pulse energies (3.8 and $2.0 \mu \mathrm{J}$ ). Each data point shown in Figure $8 \mathrm{a}$ and $\mathrm{b}$ represents the total fluorescence in a time bin of $20 \mathrm{~s}$ (200 pulses) taken throughout a $10 \mathrm{~m}$ exposure. Decay of the parent ion abundance was also measured for exposure to pulses of $2.0 \mu \mathrm{J}$ energy and is shown in Figure 8c.

The fraction of the parent ion fragmented per laser pulse was obtained by fitting the expression for $N_{m}$ given by eq 5 to parent ion decay data and the fluorescence data was fit to the expression for $\left\langle N_{h v}\right\rangle$ in eq 7 . The values of $\Gamma_{F}$ for both ion and fluorescence decays are listed in Table 2 as well as related parameters $k_{F}$ and $\langle\delta V / V\rangle_{N}$. The intensity dependence of $\Gamma_{F}$ depends on a factor $I / I_{s} /\left(1+2 I / I_{s}\right)$ which can be calculated by estimating $I_{s}=\left(h v / \sigma_{a b s} \tau_{f}\right) \approx 3 \mathrm{MW} / \mathrm{cm}^{2}$ for $\sigma_{a b s} \approx 1.5 \AA^{2}$ [25] at $355 \mathrm{~nm}$ and $\tau_{f} \approx 3 \mathrm{~ns}$. The ratio of intensity factors for $3.8 \mu \mathrm{J}\left(1.4 \mathrm{MW} / \mathrm{cm}^{2}\right)$ and $2.0 \mu \mathrm{J}(0.74 \mathrm{MW} /$ $\mathrm{cm}^{2}$ ) is just 1.41 , which compares closely with the experimental value $\Gamma_{F}=1.38$. The photofragmentation rate constant was identical for both intensities within the experimental uncertainty, which suggests that multiphoton excitation processes were not important in these experiments.

The parent ion decay parameters obtained for $2.0 \mu \mathrm{J}$

Table 1. Temperature variation of parent ion decay

\begin{tabular}{lccccrc}
\hline Decay & $E(\mu \mathrm{J})$ & $q_{z}$ & $T(K)$ & $\langle\delta V / V\rangle_{N}{ }^{a}$ & $\Gamma_{F}$ & $k_{F}\left(s^{-1}\right)$ \\
\hline \hline lon & 2 & 0.46 & 300 & 0.049 & $(1.84 \pm .1) \times 10^{-3}$ & $1.5 \times 10^{7}$ \\
lon & 2 & 0.46 & 140 & 0.068 & $(7.2 \pm .9) \times 10^{-4}$ & $4.1 \times 10^{6}$ \\
\hline
\end{tabular}

aThe volume fraction $\langle\delta V / V\rangle_{N}$ was averaged over ion number and used in eq 6 to determine $k_{F}$. 


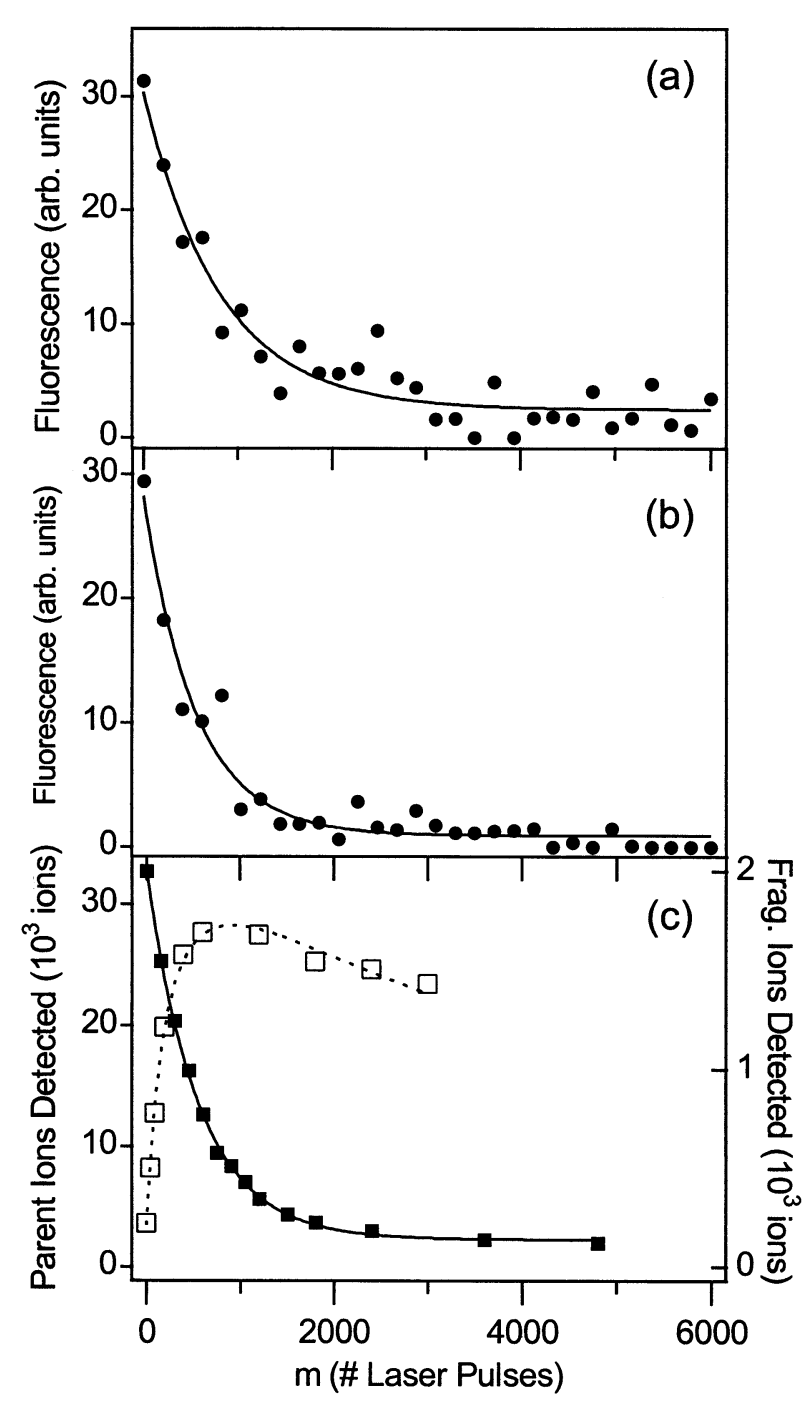

Figure 8. Fluorescence from Alexa Fluor350 (filled circle) shows a decay in intensity during an extended exposure $(10 \mathrm{~min})$ at two pulse energies, (a) $2 \mu \mathrm{J} /$ pulse and (b) $3.8 \mu \mathrm{J} /$ pulse. Each data point represents the total fluorescence in a time bin of $20 \mathrm{~s}$ (200 pulses) from a $10 \mathrm{~min}$ exposure (c). The Parent ion number (filled square) shows an identical decay rate and is accompanied by a growth of the fragment ions (open square). The solid curves are exponential fits to the data. All data were taken at $q_{z}=0.61$ and $T=300 \mathrm{~K}$.

pulses are shown in Table 2. To compare the parent ion decay fraction per pulse and the fragmentation rate with those parameters obtained for fluorescence decay, it is necessary to explicitly include the variation of $\delta V / V$ with $N_{m}$ in the preexponential factor of eq 7. This factor is calculated as a function of ion number as described in Appendix A1 and included in the fluorescence fit by scaling the initial fluorescence value in Figure 8a with the initial ion number in Figure 8c. Both ion and fluorescence data were taken during the same experimental run where ion loading reproducibility is $\geq 90 \%$. The parameter $\Gamma_{F}$ derived by fitting fluorescence data to eq 7 is included in Table 2 and agrees with $\Gamma_{F}$ of the ion decay data to within experimental uncertainty. This not only reinforces the basis for fluorescence decay, but also serves to check the consistency of this relatively simple fragmentation model.

These experiments were not designed to relate fragmentation rates, $k_{F}$, to molecular processes. The most important uncertainty is the level of internal energy deposited in the molecule prior to dissociation. This is not only a consequence of the unknown quantum yield, but also the possibility for exposure of a molecule to multiple laser pulses. The measured mass of the fragment suggests that the molecule dissociates upon breaking a $\mathrm{C}-\mathrm{O}$ bond, which can require an energy of $\sim 3 \mathrm{eV}$. For such a bond strength, an average internal energy of up to $\sim 20 \mathrm{eV}$ would be required to yield the measured rates of $k_{F} \sim 10^{6}-10^{7} \mathrm{~s}^{-1}$. It is not clear from these experiments just how the ion-radiative interaction provides for the accumulation of such internal energy. It may be possible that the dissociation is preferentially occurring for ions having trajectories which keep them within the interaction volume, so that multiple exposure of a given ion becomes more probable. Another contribution can arise from molecular kinetic energy which is converted to internal energy via collisions with the He background gas. A better description of this infrequent fragmentation process (only $\sim 0.1 \%$ per pulse) will require more investigation of the dependence on ion trap parameters including background gas pressure, and also laser parameters such as pulse repetition rate and fluence.

\section{Summary and Conclusions}

Laser-induced fluorescence measurements on Rhodamine 640 and Alexa Fluor 350 molecular ions stored in an rf quadrupole trap have demonstrated high detection sensitivity achieved by eliminating background laser scattering during the pulse. Measurements of $\sim 100$ molecular ions could be performed during $\sim 3$

Table 2. Intensity variation of fluorescence decay and comparison with parent ion decay

\begin{tabular}{lcccccc}
\hline Decay & $E(\mu \mathrm{J})$ & $q_{z}$ & $T(K)$ & $\langle\delta V / V\rangle_{N}{ }^{a}$ & $\Gamma_{F}$ & $k_{F}\left(s^{-1}\right)$ \\
\hline \hline lon & 2 & 0.61 & 300 & 0.078 & $(1.79 \pm .04) \times 10^{-3}$ & $9.3 \times 10^{6}$ \\
Fluor & 2 & 0.61 & 300 & 0.078 & $(1.25 \pm .15) \times 10^{-3}$ & $6.5 \times 10^{6}$ \\
Fluor & 3.8 & 0.61 & 300 & 0.078 & $(1.85 \pm .1) \times 10^{-3 b}$ & $9.6 \times 10^{6}$ \\
\hline
\end{tabular}

aThe volume fraction $\langle\delta V / V\rangle_{N}$ was averaged over ion number and used in eq 6 to determine $k_{F}$.

${ }^{b}$ Fluorescence decay at $2 \mu \mathrm{J}$ follows from an analysis which includes the variation of $\delta V / V$ with ion number in the preexponential factor of eq 7. 
min exposures. Photon statistics were determined from an analysis of $10 \mathrm{~m}$ exposures of $\sim 1000$ ions within the ion cloud volume overlapped by the laser beam. This analysis indicated that under these conditions, an average of $\sim 10$ photons were incident on the PMT during each 15 ns laser pulse. This result was consistent with an estimate of the detected fluorescence from the known radiative parameters, solid angle and interaction volume.

The variation of fluorescence intensity with trapped ion number clearly displayed a nonlinear dependence that was consistent with saturation of the ion density as a result of space charge electrostatic repulsion. Scanning the detection optics across the trap output aperture provided a method to optimize the detected signal along both cloud directions. As a result, the fluorescence intensities obtained were sensitive to the peak ion cloud density. The ion density was calculated selfconsistently within a mean field approximation and these calculations accurately reproduce the ion number dependence exhibited by the fluorescence data taken at different trap well depths and ion kinetic temperatures. These experimental trap parameters, together with ion number, characterized the competition between the trapping potential and the space charge potential which determines the cloud density.

Photofragmentation of Alexa Fluor 350 was measured by the decay of fluorescence emission and also by the decay of the parent ion number at different temperatures and trapping parameters. The data were fit to a rate equation model that accounted for the small interaction volume within a reservoir of unexposed molecules. These data were described remarkably well by the dynamic processes incorporated in the model and demonstrate the quantitative capability of these fluorescence techniques when the effects of space charge effects are accounted for.

In summary, these fluorescence measurements presented clear evidence of emission from an ion cloud having a space charge limited density. Aside from this important characteristic, fluorescence measurements were straightforward and readily interpreted. These results suggest that the techniques described here will be useful to apply fluorescence measurements as an in situ probe of trapped ion properties including species identity, ion spectroscopy, and the growth and decay dynamics of specific ion populations. We have recently started experiments to monitor changes in the conformation of biopolymer ions by measuring the FRET between fluorophores attached to the ends of a DNA double strand. These experiments will measure the correlation between in situ fluorescence data and ion mass spectra as a function of temperature to investigate intermediate states leading to dissociation of the double strand.

Improvements of the detection optical configuration will replace glass filters by dielectric filters (Chroma Technology Corp., Brattleboro, VT) which attenuate by o.d. 6 at the laser wavelength. In addition, the lens system will be changed to a confocal design that will greatly reduce aberration losses at the PMT aperture. Various different trap technologies are also being considered. Fluorescence detection of ions stored in a linear quadrupole [26] would reduce the laser scattering from trap electrodes yet provide fluorescence collection from a larger laser-cloud interaction volume. Cylindrical ion traps $[27,28]$ offer the possibility to detect through a ring electrode formed from highly transparent $(\sim 80 \%)$ $\mathrm{Ni}$ screen. This design would also provide fluorescence collection from a significantly greater solid angle as shown in reference [29] and increase the trap dimensions to reduce laser background scattering.

\section{Acknowledgments}

This work is supported by The Rowland Institute for Science. The authors acknowledge helpful discussions with Dr. James Foley, Dr. Detlef Schooss, and Dr. Michael Burns and thank Dr. Stefan Krückeberg for helping with the data acquisition software.

\section{Appendix: Fluorescence Analysis for Trapped Ions}

\section{A. Fluorescence from Space Charge Limited Ion Density}

The following fluorescence model is derived under the assumption that the ion cloud can be represented as a diffusive gas without including the long range correlations describing the trajectory of each trapped ion. This model will not be appropriate to describe physical processes which occur predominantly for specific ensembles of trajectories. For example, measurements of the photofragmentation of trapped ions will probe only those ions which have trajectories which spend sufficient time within the laser-ion cloud interaction volume. As will be shown below, such considerations can be important for interpretation of photofragmentation data.

The fluorescence of trapped ions will be modeled by rate equations that include processes shown schematically in Figure 9. In this approximation, vibrational relaxation is assumed to be sufficiently rapid to represent the radiative interaction as occurring between two levels, the ion ground state $|g\rangle$ and excited state $|e\rangle$ having energy separation hv. The ion populations in these states, $n_{g}$ and $n_{e}$ respectively, interact with the laser intensity, $I$, with an absorption cross-section $\sigma_{a b s}$. The photon absorption (and emission) rate is indicated by $\left(\sigma_{a b s} I / h v\right)$ and and $\tau_{f}$ is the fluorescence lifetime.

The time dependence of the population densities within the overlap volume of these states are described by the following rate equations

$$
\begin{aligned}
& \frac{d n_{e}}{d t}=n_{g} \frac{\sigma_{a b s} I}{h v}-n_{e}\left(\frac{\sigma_{a b s} I}{h v}+\frac{1}{\tau_{f}}\right) \\
& \frac{d n_{g}}{d t}=-n_{g} \frac{\sigma_{a b s} I}{h v}+n_{e}\left(\frac{\sigma_{a b s} I}{h v}+\frac{1}{\tau_{f}}\right) .
\end{aligned}
$$




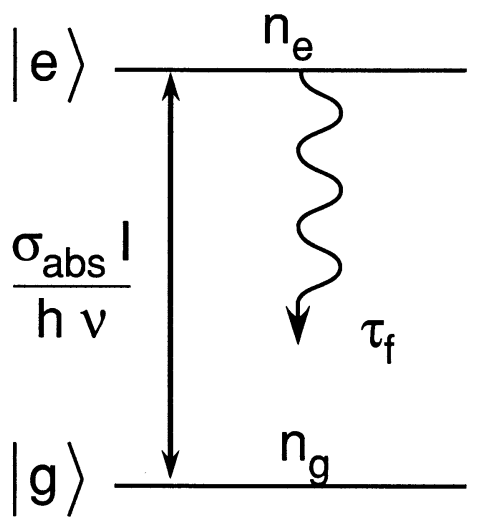

Figure 9. Schematic energy level diagram for an ion transition between ground $(g)$ and excited $(e)$ states separated by energy $h v$.

The total ion density in the interaction volume, $n=n_{e}+$ $n_{g}$ is just the trapped ion cloud density since ion trajectories refill this volume with unexposed ions during the $100 \mathrm{~ms}$ between laser pulses. Since this analysis assumes the absence of any process which involves a decay of the parent ion, the total ion population is given by $n=n_{g}+n_{e}$. The solution to eqs A1 and A2 for the excited state population is

$$
\begin{aligned}
& n_{e} \approx n \frac{I / I_{S}}{1+2 I / I_{S}}\left(1-e^{-\gamma^{\prime} t}\right) 0 \leq t \leq \tau_{p} \\
& \text { where } \gamma^{\prime}=\frac{2 \sigma_{a b s} I}{h v}+\frac{1}{\tau f}
\end{aligned}
$$

This solution includes the saturation of the radiative transition by the laser intensity, I, which acts to reduce fluorescence by stimulating a transition to the ground state. For the ion-laser interactions under consideration here, $\tau_{p} \gg \tau_{f} \approx\left(h v / \sigma_{a b s \mathrm{I} I}\right)$, and $\gamma^{\prime} \tau_{p} \gg 1$ for which the parenthetical expression in eq A3 is approximately unity. The density of excited state ions, $n_{e}$, is then given by

$$
n_{e}=n \frac{I / I_{S}}{1+2\left(I / I_{S}\right)}
$$

where $I_{S}$ is the saturation intensity, $I_{S} \equiv h v / \sigma_{a b s} \tau_{f}$, characterizing the radiative transition by the absorption cross-section, $\sigma_{a b s}$ and the radiative lifetime. Note that the maximum excited state density approaches $n / 2$ as the intensity becomes $I \gg I_{S}$.

Space charge ion density. A self-consistent treatment of the space charge interaction in ion clouds is required in order to extend ion motion calculations to higher densities of current experimental interest. For large clouds of $N \geq 10^{4}$ ions, the rigorous approach of introducing sums over Coulomb interacting ion pairs is both limited by calculation runtimes and probably not required if only average cloud physics is of interest. The following mean field treatment explicitly includes a calculation of the space charge potential to derive the ion motion self consistently. However, the following approximation is limited to ion motion for which the secular motion [23] dominates the ion trajectory $\left(q_{z} \leq 0.4\right)$.

In thermal equilibrium, the ion density can be expressed by

$$
n(r, z)=n_{p} \exp [-e \Phi(r, z) / k T]
$$

in which the total potential experienced by an ion is assumed to be a sum of the trap potential and a space charge potential $\Phi=\Phi_{T}+\varphi_{s c}$ and the peak ion density $n_{p}$ is defined below. Poisson's equation $\nabla^{2} \varphi_{s c}(r, z)$ can then be solved numerically for $\varphi_{s c}$ using eq A5.

In this paper, space charge calculations were primarily used to demonstrate the correlation between the fluorescence data and peak ion density as shown in Figure 4. In addition, calculation of the density spatial distribution provided estimates of the laser-ion cloud interaction volume $\delta V$ and also the volume fraction, $\delta V / V$, as a function of $N$.

The interaction volume can be defined by considering the ion density which is exposed to a laser propagating in the $\hat{y}$ direction along the radial axis of the cloud and focussed to a cross-sectional area $A$. Derivations performed below will require an estimate for the number of ions within the interaction volume. Assuming that the laser cross-section is sufficiently small that the variation of $n$ is slow over this area, the density-volume integral over $\delta V$ can be approximated by

$$
\begin{aligned}
\iiint_{\delta V} n d^{3} v & \approx \iiint_{\delta v} n_{p} f(x, y, z) d A d y \\
& \approx n_{p} A \int_{-r_{0}}^{r_{0}} f(0, y) d y \\
& \approx n_{p} A 2 \sigma_{r}
\end{aligned}
$$

where the length of the laser-ion interaction volume, $\delta V=A 2 \sigma_{r}$, is given by $\sigma_{r}$, the HWHM of the ion cloud distribution. The interaction volume is a slowly varying function of $N$, increasing with $N$ as the cloud expands by ion-ion interactions. For example, space charge calculations indicate that the interaction volume increases only by a factor of $\sim 2-3$ as $N$ varies by a factor of $10-15$ as shown in Figure 10a. For the range of $N$ involved in the experiments described here, the interaction volume was $\delta V \sim 10^{-5} \mathrm{~cm}^{-3}$. The ion cloud volume, defined here by $V \equiv N / n_{p}$, also slowly increases with $N$ but faster than $\delta V$ so that the volume fraction $\delta V / V$ decreases with $N$. Space charge calculations shown in Figure 10b indicate that the volume fraction also decreases by a factor of $\sim 2$ as $N$ increases by a factor of $10-15$. For the range of $N$ involved in the 

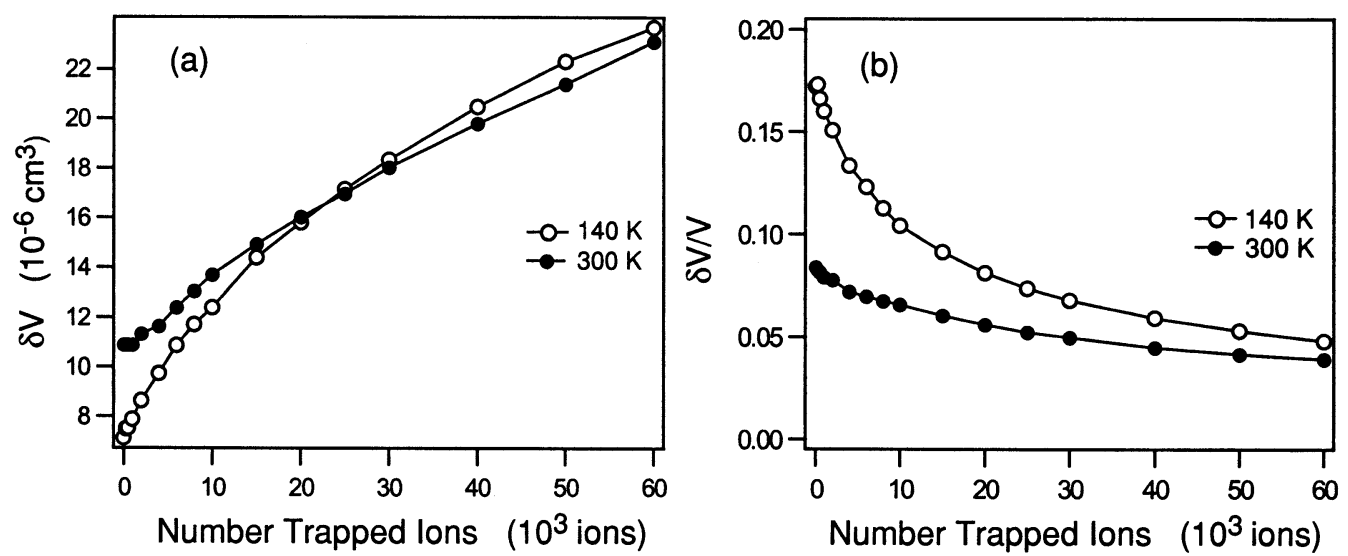

Figure 10. Space charge calculations of (a) laser-ion cloud interaction volume and (b) volume fraction versus trapped ion number calculated for Alexa Fluor350 ions at $q_{z}=0.46$, and cloud temperatures 300 and $140 \mathrm{~K}$.

experiments described here, the volume fraction was $\delta V / V \sim 0.03-0.15$. These spatial distributions were calculated for Alexa Fluor 350 ions at $q_{z}=0.46$, and cloud temperatures 300 and $140 \mathrm{~K}$.

Number of photons per laser pulse. The average number of photons per laser pulse can be obtained by considering the power radiated into a solid angle $\delta \Omega$ during the laser pulse by a density of excited state ions, $n_{e}$, within the interaction volume $\delta V$

$$
P_{\text {rad }} \iiint_{\delta V} n_{e} d^{3} v \frac{h v \delta \Omega}{\tau_{f} 4 \pi} Q Y
$$

where $h v$ is the photon energy, $\tau_{f}$ the fluorescence lifetime, and QY the fluorescence quantum yield. The fluorescence energy incident on the detector during a laser pulse of length $\tau_{p}$ is

$$
E_{\text {rad }}=\int P_{\text {rad }} d t \cdot T(1-R)
$$

where the transmission and reflectance of the detection optics are expressed by $T$ and $R$, respectively.

The average number of photons incident on the PMT detector for a single laser pulse is approximated by combining eq A4, with eqs A6-A8 to yield

$$
\left\langle N_{h v}\right\rangle=\left[\frac{n_{p} \delta V}{\tau_{f}} \frac{I / I_{S}}{1+2\left(I / I_{S}\right)} \frac{\delta \Omega}{4 \pi} Q Y\right] \tau_{p} T(1-R)
$$

which indicates the photon emission rate into solid angle $\delta \Omega$ in brackets. An estimate for $\left\langle N_{h v}\right\rangle$ can be obtained by evaluating eq A9 using a calculation for the space charge limited ion density, $n$, described above in Appendix A1. A peak ion density of $n_{p} \approx$
$2 \times 10^{8 \mathrm{~cm}^{-3}}$ is calculated for $N \approx 3 \times 10^{4}$ Rhodamine 640 ions trapped at $q_{z}=0.61$ and $T=300 \mathrm{~K}$. The interaction volume is estimated from $\delta V \approx A 2 \sigma_{r}$ for a beam focal diameter $d \approx 150 \mu \mathrm{m}$ and HWHM of the radial cloud distribution $\sigma_{r} \approx 0.04 \mathrm{~cm}$. In addition, using parameters appropriate for these experimental conditions $I / I_{s} \approx 1.7, \delta \Omega / 4 \pi \approx 5 \times 10^{-3}, \tau_{f}=3.5 \mathrm{~ns}$, $\tau_{p} \approx 15 \mathrm{~ns}, Q Y \sim 0.95$ [30], and $T(1-R) \approx 0.26$ yields $\left\langle N_{h v}\right\rangle \approx 6$ photons per laser pulse.

\section{B. Photon Statistics}

Fluctuations in the fluorescence signal displayed in Figure 4 indicate that a large fraction of laser pulses did not result in detected photoelectrons. These fluctuations in the number of photoelectrons generated by the fluorescence pulses can be analyzed by calculating the statistics of the number of detected photons [31]. Assuming the probability that $\mathrm{m}$ photons will strike the PMT during a laser pulse is described by a Poisson distribution, $P_{m}$

$$
P_{m}=\frac{\mu^{m}}{m !} e^{-\mu},
$$

and $P_{r, m}$ is the probability of producing $r$ photoelectrons given $m$ incident photons and given by

$$
P_{r, m}(\eta)=\eta^{r}(1-\eta)^{m-r} \frac{m !}{r !(m-r) !}
$$

the fraction of data pulses resulting in $r$ photoelectrons is estimated by

$$
P_{r}(\eta)=\sum_{m} P_{m} P_{r, m}(\eta)=\frac{(\mu \eta)^{r}}{r !} e^{-\mu \eta}
$$

where $\eta$ is the PMT quantum efficiency and $\mu$ is the average number of photons striking the PMT during each pulse. Note that the probability for $r=0, P_{0}(\eta)=$ 
$e^{-\mu \eta}$, is directly determined from the fraction of zero amplitude pulses ocurring in the data. For the example shown in Figure $4, P_{0}(\eta)=0.38$, and this yields an average number of photons per pulse incident on the PMT, of $\mu \approx 10$ photons for $\eta \approx 0.1$ at the fluorescence peak of Rhodamine 640 . Having determined the product $\mu \eta$, the photoelectron distribution $P_{r}(\eta)$ can be calculated as a function of photoelectron number and is shown in Figure 5.

\section{Reservoir Photofragmentation Model}

The following model is derived under the assumption that the ion cloud can be represented as a diffusive gas without including the long range correlations describing the trajectory of each trapped ion. This model will not be appropriate to describe physical processes which occur predominantly for specific ensembles of trajectories. The photofragmentation of trapped ions will be modeled by rate equations that include the processes shown schematically in Figure10 but including an additional excited state decay channel characterizing photofragmentation with a rate $k_{F}$. Although this rate usually characterizes a unimolecular dissociation process following the deposition of a known energy into the internal degrees of freedom, we use this rate here to describe an average dissociation rate which depends on the laser parameters and also the ion trap parameters.

In the prescence of photofragmentation, the time dependence of the population densities within the overlap volume are described by the following rate equations

$$
\begin{aligned}
& \frac{d n_{e}}{d t}=n_{g} \frac{\sigma_{a b s} I}{h v}-n_{e}\left(\frac{\sigma_{a b s} I}{h v}+\frac{1}{\tau_{f}}+k_{F}\right) \\
& \frac{d n_{g}}{d t}=-n_{g} \frac{\sigma_{a b s} I}{h v}+n_{e}\left(\frac{\sigma_{a b s} I}{h v}+\frac{1}{\tau_{f}}\right) .
\end{aligned}
$$

Ion trajectories refill the overlap region in a time $(\sim 10$ $\mu \mathrm{s})$ short compared to the $100 \mathrm{~ms}$ between laser pulses. In addition, the fragment ions do not contribute to the trapped ion density since they are unstable under our operating conditions. As a result, the total ion density, $n(t)=n_{e}+n_{g}$, within the interaction volume is equal to the trapped density at any time. Although the rates of change of $n_{e}$ and $n_{g}$ are dominated by radiative rates, $\left(\sigma_{a b s} \mathrm{I} / \mathrm{hv}\right) \gg k_{F}$, photofragmentation accounts for the slow decay the trapped density

$$
\frac{d n}{d t}=\frac{d n_{e}}{d t}+\frac{d n_{g}}{d t}=-k_{F} n_{e}
$$

A solution for $n_{e}$ is obtained from eq A13 by substituting $N_{g}=n-n_{e}$ and assuming that $n$ is approximately constant during the radiative interaction. The solution for the excited state population is

$$
n_{e} \approx n \frac{I / I_{S}}{1+2\left(I / I_{S}\right)}\left(1-e^{-\gamma^{\prime} t}\right) \quad 0 \leq t \leq \tau_{p}
$$

where $\gamma^{\prime}=2 \sigma \mathrm{absIhv}+1 \tau \mathrm{f}+\mathrm{kF}$. For the ion-laser interactions under consideration here, $\tau_{p} \gg \tau_{f} \approx(h v /$ $\left.\sigma_{a b s} \mathrm{I}\right)$, and $\gamma_{p}^{\prime} \gg 1$ for which the final parenthetical expression in eq A16 is approximately unity.

To consider the rate of change of the total number of trapped parent ions, $N$, it will be necessary to consider only those ions in the interaction volume given by the volume integrals

$$
\frac{d N}{d t}=\frac{d}{d t} \iiint n d^{3} v=-k_{F} \iiint_{\delta V} n_{e}(N) d^{3} v
$$

The last integral is limited to the interaction volume since the excited state ions will decay $\left(\tau_{f} \sim 3 \mathrm{~ns}\right)$ before their trajectories leave this volume. Expressing the density of excited state ions, $n_{e}$, by eq A16 yields the change in ion number caused by the $m$ th laser pulse

$$
\begin{aligned}
\Delta N & =N_{m+1}-N_{m} \\
& =-k_{F} \frac{I / I_{S}}{1+2\left(I / I_{S}\right)} \tau_{p} \iiint_{\delta V} n\left(N_{m}\right) d^{3} v .
\end{aligned}
$$

The laser-ion interaction volume is sufficiently small that the variation of $n\left(N_{m}\right)$ is slow over $\delta V$ and the volume integral in eq A18 can be approximated similar to eq A6 as

$$
\iiint_{\delta V} n d^{3} v \approx n_{p} \delta V \equiv N_{m} \frac{\delta V}{V}
$$

where the interaction volume and the ion cloud volume are defined above. In this approximation, the difference equation governing the ion decay for $\delta m=1$

$$
\Delta N=N_{m+1}-N_{m}=-k_{F} \frac{I / I_{S}}{1+2\left(I / I_{S}\right)} \tau_{p} N_{m} \frac{\delta V}{V}
$$

characterizes an exponential decay of the parent ion given by

$$
N_{m}=N_{0} \exp \left(-\Gamma_{F} m\right)
$$

where

$$
\Gamma_{F}=k_{F} \tau_{p} \frac{I / I_{S}}{1+2\left(I / I_{S}\right)} \frac{\delta V}{V}
$$

is just the fraction of the parent ion fragmented per laser pulse. For experiments described here, $\Gamma_{F} \sim 10^{-3}$ de- 
pending on trapping parameters and ion cloud temperature.

The decay of the fluorescence signal resulting from photofragmentation follows from eq A9 giving the number of photons detected from the interaction volume $\delta V$

$$
\left\langle N_{h v}\right\rangle=\text { constant } \cdot n_{e} \delta V \approx \text { constant } \cdot N_{m} \frac{\delta V}{V} .
$$

The decay of the fluorescence is then given by

$$
\left\langle N_{h v}\right\rangle \approx \text { constant } \cdot N_{0} \frac{\delta V}{V} \exp \left(-\Gamma_{F} m\right) .
$$

In order to extract the decay parameter $\Gamma_{F}$ by fitting data with eqs A21 and A24, the volume fraction $\delta \mathrm{V} / \mathrm{V}$ will be approximated by a constant average value. Since the volume fraction increases slowly as the trapped ion number decreases as shown above in Figure 10b, this approximation will introduce a small uncertainty in $\Gamma_{F}$. However, to obtain a consistent comparison between $\Gamma_{F}$ derived from decay of the parent ion and that obtained from the fluorescence data, the ion number dependence of the preexponential factor $\delta V / V$ in eq A24 will have to be included in fitting fluorescence data.

\section{References}

1. Bialas, J.; Blatt, R.; Neuhauser, W.; Toschek, P. E. Ultrasensitive Detection of Light Absorption by Few Ions. Opt. Commun. 1986, 59, 27-30.

2. Bergquist, J. C.; Wineland, D. J.; Itano, W. M.; Hemmati, H.; Daniel, H.-U.; Leuchs, G. Energy and Radiative Lifetime of the $5 \mathrm{~d}^{9} 6 \mathrm{~s}^{2}{ }^{2} \mathrm{D}_{5 / 2}$ state in $\mathrm{Hg}$ ii by Doppler-Free Two-Photon Laser Spectroscopy. Phys. Rev. Lett. 1985, 55, 1567-1570.

3. Plumelle, F.; Desaintfuscien, M.; Duchene, J. L.; Audoin, C. Laser Probing of Ions Confined in a Cylindrical Radiofrequency Trap. Opt. Commun. 1980, 34, 71-76.

4. Blatt, R.; Werth, G. Precision Determination of the GroundState Hyperfine Splitting in ${ }^{137} \mathrm{Ba}^{+}$Using the Ion-Storage Technique. Phys. Rev. 1982, A25, 1476-1482.

5. Danon, J.; Mauclaire, G.; Govers, T. R.; Marx, R. Laser Induced Fluorescence of Ions Trapped in an Ion Cyclotron Resonance Cell: Excitation of $\mathrm{CO}^{+} \chi^{2} \Sigma, \nu^{\prime \prime}=0$ and relaxation of $\mathrm{CO}^{22} A^{2} \Pi$, $\nu^{\prime}=1$. J. Chem. Phys. 1982, 76, 1255-1262.

6. Grieman, F. J.; Mahan, B. H.; O'Keefe, A. The Laser Induced Fluorescence Spectrum of Trapped $\mathrm{CD}^{+}$. J. Chem. Phys. 1980, $72,4246-4247$.

7. Martner, C. C.; Pfaff, J.; Rosenbaum, N. H.; O'Keefe, A.; Saykally, R. J. Radiative Lifetimes of Trapped Molecular Ions: $\mathrm{HCl}^{+}$and $\mathrm{HBr}^{+}$. J. Chem. Phys. 1983, 78, 7073-7076.

8. Wang, Y.; Hendrickson, C. L.; Marshall, A. G. Direct Optical Spectroscopy of Gas-Phase Molecular Ions Trapped and MassSelected by Ion Cyclotron Resonance: Laser-Induced Fluorescence Excitation Spectrum of Hexafluorobenzene $\left(\mathrm{C}_{6} \mathrm{~F}_{6}^{+}\right)$. Chem. Phys. Lett. 2001, 334, 69-75.

9. Neuhauser, W.; Hohenstatt, M.; Toschek, P.; Dehmelt, H. Optical-Sideband Cooling of Visible Atom Cloud Confined in Parabolic Well. Phys. Rev. Lett. 1978, 41, 233-236.
10. Diedrich, F.; Peik, E.; Chen, J. M.; Quint, W.; Walther, H. Observation of a Phase Transition of Stored Laser-Cooled Ions. Phys. Rev. Lett. 1987, 59, 2931-2934.

11. Wineland, D. J.; Bergquist, J. C.; Itano, W. M.; Bollinger, J. J.; Manney, C. H. Atomic Ion Coulomb Clusters in an Ion Trap. Phys. Rev. Lett. 1987, 59, 2935-2938.

12. Hornekær, L.; Kjærgaard, N.; Thommesen, A. M.; Drewsen, M. Structural Properties of Two-Component Coulomb Crystals in Linear Paul Traps. Phys. Rev. Lett. 2001, 86, 1994-1997.

13. Jarrold, M. F. Peptides and Proteins in the Vapor Phase. Annu. Rev. Phys. Chem. 2000, 51, 179-207.

14. Selvin, P. R. The Renaissance of Fluorescence Resonance Energy Transfer. Nature Struct. Biol. 2000, 7, 730-734.

15. Schnier, P. D.; Klassen, J. S.; Strittmatter, E. F.; Williams, E. R. Activation Energies for Dissociation of Double Strand Oligonucleotide Anions: Evidence for Watson-Crick Base Pairing in Vacuo. J. Am. Chem. Soc. 1998, 120, 9605-9613.

16. Gabryelski, W.; Li, L. Photo-Induced Dissociation of Electrospray Generated Ions in an Ion Trap/Time-of-Flight Mass Spectrometer. Rev. Sci. Instrum. 1999, 70, 4192-4199.

17. Creaser, C. S.; O'Neill, K. E. Photodissociation and Collisionally Activated Dissociation Tandem Mass Spectrometric Studies of Difluoro[triazol-1-ylmethyl]Benzhydrols and Related Compounds in a Quadruple Ion trap. Int. J. Mass Spectrom. Ion Processes 1997, 165, 13-23.

18. Fenn, J. B.; Mann, M.; Meng, C. K.; Wong, S. F.; Whitehouse, C. M. Electrospray Ionization for Mass Spectrometry of Large Biomolecules. Science 1989, 246, 64-71.

19. Kebarle, P.; Tang, L. From Ions in Solution to Ions in the Gas Phase: The Mechanism of Electrospray Mass Spectrometry. Anal. Chem. 1993, 65, 972A-986A.

20. Fenn, J. B.; Mann, M.; Meng, C. K.; Wong, S. F.; Whitehouse, C. M. Electrospray Ionization-Principles and Practice. Mass Spectrom. Rev. 1990, 9, 37-70.

21. Parks, J. H.; Pollack, S.; Hill, W. Cluster Experiments in Radio Frequency Paul traps: Collisional Relaxation and Dissociation. J. Chem. Phys. 1994, 101, 6666-6685.

22. Ferreira, J. A. B.; Costa, S. M. B. Rhodamine 3B+ $\mathrm{ClO} 4-$ Electronic Transitions: Reaction Field and Vibrational Structure. Chem. Phys. 2001, 273, 39-49.

23. Parks, J. H.; Szöke, A. Simulation of Collisional Relaxation of Trapped Ion Clouds in the Presence of Space Charge Fields. J. Chem. Phys. 1995, 103, 1422-1439.

24. Guan, S.; Marshall, A.G. Equilibrium Space Charge Distribution in a Quadrupole Ion Trap. J. Am. Soc. Mass Spectrom. 1994, $5,64-71$.

25. Molecular Probes Home Page. http://www.probes.com/ media/pis/mp10168.pdf, (accessed October, 2001).

26. Welling, M.; Schuessler, H. A.; Thompson, R. I.; Walther, H. Ion/Molecule Reactions, Mass Spectrometry, and Optical Spectroscopy in a Linear Ion Trap. Int. J. Mass Spectrom. Ion Processes 1998, 172, 95-114.

27. Wells, J. M.; Badman, E. R.; Cooks, R. G. A Quadrupole Ion Trap with Cylindrical Geometry Operated in the Mass-Selective Instability Mode. Anal. Chem. 1998, 70, 438-444.

28. Arkin, C. R.; Goolsby, B.; Laude, D. A. Characterization of a Hybrid Ion Trap. Int. J. Mass Spectrom. 1999, 190, 47-57.

29. Mahan, B. H.; O'Keefe, A. Rotational Excitation and Radiative Lifetimes of $\mathrm{N}_{2}^{+}$. J. Chem. Phys. 1981, 74, 5606-5612.

30. Beaumont, P. C.; Johnson, D. G.; Parsons, B. J. Photophysical Properties of Laser Dyes: Picosecond Laser Flash Photolysis Studies of Rhodamine 6G, Rhodamine B, and Rhodamine 101. J. Chem. Soc. Faraday Transactions 1993, 89, 4185-4191.

31. Engstrom, R. W. Photomultiplier Handbook. RCA Solid State Division, Electro Optics and Devices: Lancaster, 1980; 160163. 